\title{
Security Notions for Information Theoretically Secure Encryptions
}

\author{
Mitsugu Iwamoto \\ Center for Frontier Science, \\ the University of Electro-Communications \\ 1-5-1 Chofugaoka, Chofu-shi, Tokyo, 182-8585 Japan \\ Email: mitsugu@inf.uec.ac.jp
}

\author{
Kazuo Ohta \\ Graduate School of Informatics and Engineering, \\ the University of Electro-Communications \\ 1-5-1 Chofugaoka, Chofu-shi, Tokyo, 182-8585 Japan \\ Email: ota@inf.uec.ac.jp
}

\begin{abstract}
This paper is concerned with several security notions for information theoretically secure encryptions defined by the variational (statistical) distance. To ensure the perfect secrecy (PS), the mutual information is often used to evaluate the statistical independence between a message and a cryptogram. On the other hand, in order to recognize the information theoretically secure encryptions and computationally secure ones comprehensively, it is necessary to reconsider the notion of PS in terms of the variational distance. However, based on the variational distance, three kinds of definitions for PS are naturally introduced, but their relations are not known. In this paper, we clarify that one of three definitions for PS with the variational distance, which is a straightforward extension of Shannon's perfect secrecy, is stronger than the others, and the weaker two definitions of PS are essentially equivalent to the statistical versions of indistinguishability and semantic security.
\end{abstract}

\section{INTRODUCTION}

Perfect secrecy (PS) is a strong security notion which is secure against an adversary with unbounded computing power. Perfect secrecy was defined by Shannon [1], and he proved that perfect secrecy is achieved by one time pad (Vernam) cipher [2]. Furthermore, in order to achieve perfect secrecy, Shannon also proved in [1] that the entropy of a key must be greater than the entropy of a message, which makes perfect secrecy quite impractical.

Roughly speaking, PS is defined by the statistical independence between a message $M$ and a cryptogram $C$. Specifically, we often require almost statistical independence between $C$ and $M$ to ensure PS. We note here that two metrics can be used to measure the almost statistical independence, i.e., the mutual information and the variational (statistical) distance. In general, the mutual information is often used in information theoretic cryptography since it guarantees stronger security compared to the security notions based on the variational distance due to Pinsker's inequality. On the other hand, the variational distance is often used in computationally secure cryptography: For instance, indistinguishability (IND) and semantic security (SS) are defined in terms of the variational distance. We note that several researchers recently discussed one time pad cipher under the security notions developed in computationally secure cryptography. For instance, RussellWang [3] introduced entropic security based on semantic security, and they succeeded in shortening the key length of a symmetric key cryptosystem which is secure against an unbounded adversary. In addition, Dodis-Smith [4] introduced another security notion which is closely related to indistinguishability, and they gave the other realization of entropic security by using extractors [5].

Given the above backgrounds, we are interested in PS defined by the variational distance, and its relation to IND and SS, which will be some help for comprehensive understanding of information theoretically secure encryptions and computationally secure ones. However, as we will see in Definition 2, three kinds of definitions of PS denoted by $\mathrm{PS}_{* M}(\varepsilon), \mathrm{PS}_{C *}(\varepsilon)$, and $\mathrm{PS}_{C M}(\varepsilon)$ can be naturally introduced in terms of the variational distance. It is obvious that these three notions of PS are the same when $\varepsilon=0$. However, in the case of $\varepsilon>0$, their relations are not known. In this paper, we will point out that $\mathrm{PS}_{* M}(\varepsilon)$ is stronger than the others by showing a pathological example. Furthermore, it will be proved that the remaining two definitions $\mathrm{PS}_{C *}(\varepsilon)$ and $\mathrm{PS}_{C M}(\varepsilon)$ guarantee essentially the same security as the statistical versions of IND and SS.

The rest of this paper is organized as follows: In Section II, notations and three variations of PS are introduced. Statistical IND is introduced in section III, and the relations between PS and statistical IND are clarified. A relation between statistical IND and statistical SS is proven in Section IV. Finally, a gap between one of three variations of PS and the other security notions are pointed out in Section V. Technical lemmas are provided in Appendix.

\section{Preliminaries}

Let $M, K$, and $C$ be random variables taking values in finite sets $\mathcal{M}, \mathcal{K}$, and $\mathcal{C}$, which correspond to sets of messages, keys, and cryptograms, respectively. For a random variable $X$ taking values in a finite set $\mathcal{X}$ and an element $x \in \mathcal{X}$, denote by $P_{X}(x)$ a probability of $X=x$. Let $\mathcal{P}(\mathcal{X})$ be the totality of probability distributions over $\mathcal{X}$.

A symmetric key cryptography $\Sigma$ consists of a probability distribution $P_{K} \in \mathcal{P}(\mathcal{K})$ of a key, and a pair of an encryption function Enc : $\mathcal{M} \times \mathcal{K} \rightarrow \mathcal{C}$, and a decryption function Dec $: \mathcal{C} \times \mathcal{K} \rightarrow \mathcal{M}$, i.e., $\Sigma \stackrel{\text { def }}{=}\left(P_{K}\right.$, Enc, Dec $)$. Note that $K$ is chosen independently of a message $M$, and Enc and Dec are deterministic maps. Suppose that a message is generated 
according to a probability distribution $P_{M} \in \mathcal{P}(\mathcal{M})$. Then, the probability distribution $P_{C}$ of a cryptogram is determined by $P_{M}, P_{K}$ and Enc. Let $P_{C M}$ be a joint probability distribution of a cryptogram $C$ and a message $M$, and denote by $P_{C \mid M}$ a conditional distribution of a cryptogram when a message is given. Denote by $\mathbb{P}_{C \mid M}$ an $|\mathcal{C}| \times|\mathcal{M}|$ transition probability matrix ${ }^{1}$ associated with $\left\{P_{C \mid M}(c \mid m)\right\}_{c \in \mathcal{C}, m \in \mathcal{M}}$, i.e., each element of $\mathbb{P}_{C \mid M}$ corresponds to $P_{C \mid M}(c \mid m)$ for $c \in \mathcal{C}$ and $m \in \mathcal{M}$. The following theorem states fundamental properties of $P_{C \mid M}$ for symmetric key encryptions. The proof is provided in Appendix A.

Theorem 1: If a key $K$ is chosen independently of a message $M$, it holds that ${ }^{2}$

$$
\forall c \in \mathcal{C}, \forall m \in \mathcal{M}, P_{C \mid M}(c \mid m)=\operatorname{Pr}\{\operatorname{Enc}(m, K)=c\} .
$$

Furthermore, in the case of $|\mathcal{C}|=|\mathcal{M}|$, there exists a symmetric key cryptosystem $\Sigma$ satisfying (1) iff (if and only if) the probability transition matrix $\mathbb{P}_{C \mid M}$ is doubly stochastic ${ }^{3}$.

Hence, we assume that the conditional probability distribution $P_{C \mid M}(c \mid m), c \in \mathcal{C}, m \in \mathcal{M}$ is naturally defined by (1) if a symmetric key cryptosystem $\Sigma$ is given.

Shannon defined the notion of perfect secrecy as follows:

Definition 1 (Perfect secrecy, [1]): A symmetric key cryptosystem $\Sigma=\left(P_{K}\right.$, Enc, Dec) guarantees perfect secrecy if

$$
\forall c \in \mathcal{C}, \forall m \in \mathcal{M}, P_{M \mid C}(m \mid c)=P_{M}(m)
$$

is satisfied for arbitrary message distribution $P_{M}$.

Definition 1 means that no information of a message can be obtained from a cryptogram since a priori probability distribution $P_{M}$ of a message coincides with a posteriori probability distribution of $M$ computed by an adversary using a cryptogram.

It is easy to see that (2) is equivalent to

$$
\begin{aligned}
& \forall c \in \mathcal{C}, \forall m \in \mathcal{M}, P_{C \mid M}(c \mid m)=P_{C}(c) \\
& \forall c \in \mathcal{C}, \forall m \in \mathcal{M}, P_{C M}(c, m)=P_{C}(c) P_{M}(m)
\end{aligned}
$$

since (2) means that random variables $M$ and $C$ are statistically independent.

We are now consider relaxed definitions of perfect secrecy. That is, we define almost independence between a message $M$ and a cryptogram $C$ given by (2)-(4) in terms of the variational (statistical) distance ${ }^{4}$ denoted by $d(\cdot, \cdot)$.

Definition 2: For a real number $\varepsilon \in[0,1]$, we say that a symmetric key cryptosystem $\Sigma$ is $\mathrm{PS}_{* M}(\varepsilon)-, \mathrm{PS}_{C *}(\varepsilon)$-, or $\mathrm{PS}_{C M}(\varepsilon)$-secure if $\Sigma$ satisfies the following conditions:

$$
\begin{aligned}
\mathrm{PS}_{* M}(\varepsilon): \quad \forall P_{M} & \in \mathcal{P}(\mathcal{M}), \\
& \forall c \in \mathcal{C}, d\left(P_{M \mid C}(\cdot \mid c), P_{M}(\cdot)\right) \leq \varepsilon
\end{aligned}
$$

\footnotetext{
${ }^{1}|\cdot|$ denotes the cardinality of a set.

${ }^{2} \operatorname{Pr}\{\cdot\}$ is a probability with respect to a (joint) probability distribution of random variable(s) between the parentheses.

${ }^{3} \mathrm{~A}$ probability transition matrix $\mathbb{P}_{C \mid M}$ is doubly stochastic iff $\sum_{c \in \mathcal{C}} P_{C \mid M}(c \mid m)=\sum_{m \in \mathcal{M}} P_{C \mid M}(c \mid m)=1$ holds.

${ }^{4}$ For two probability distributions $P_{X}, P_{Y}$ over a finite set $\mathcal{A}$, the variational distance $d(\cdot, \cdot)$ is defined by $d\left(P_{X}, P_{Y}\right) \stackrel{\text { def }}{=}(1 / 2) \sum_{a \in A} \mid P_{X}(a)-$ $P_{Y}(a)\left|=\max _{f: \mathcal{A} \rightarrow\{0,1\}}\right| \operatorname{Pr}\{f(X)=1\}-\operatorname{Pr}\{f(Y)=1\} \mid$.
}

$$
\begin{gathered}
\operatorname{PS}_{C *}(\varepsilon): \quad \forall P_{M} \in \mathcal{P}(\mathcal{M}), \\
\forall m \in \mathcal{M}, d\left(P_{C \mid M}(\cdot \mid m), P_{C}(\cdot)\right) \leq \varepsilon \\
\operatorname{PS}_{C M}(\varepsilon): \forall P_{M} \in \mathcal{P}(\mathcal{M}), \\
\quad d\left(P_{C M}(\cdot \mid \cdot), P_{C}(\cdot) P_{M}(\cdot)\right) \leq \varepsilon
\end{gathered}
$$

As shown above, $\mathrm{PS}_{* M}(0), \mathrm{PS}_{C *}(0)$ and $\mathrm{PS}_{C M}(0)$ are equivalent to (2)-(4), respectively, and they are all equivalent. In this paper, we are interested in relations among these security notions when $\varepsilon$ is positive and sufficiently small. The main results of this paper are summarized as follows:

- $\mathrm{PS}_{* M}(\varepsilon)$ is the strongest among three security notions in Definition 2, which reflects the observation that $\mathrm{PS}_{* M}(\varepsilon)$ is the most straightforward extension of (2) in Definition 1.

- Two security notions in Definition 2 except for $\mathrm{PS}_{* M}(\varepsilon)$ are equivalent to each other, and they are essentially equivalent to the statistical versions of indistinguishability and semantic security which will be introduced later. As a result, it is clarified that indistinguishability and semantic security are weaker security notions even if they are formulated in information theoretically secure setting.

\section{Perfect Secrecy and Indistinguishability}

We reformulate the security notion of indistinguishability denoted by $\operatorname{IND}(\varepsilon)$ which is suitable for information theoretically secure setting. Then, we discuss the relation between $\operatorname{IND}(\varepsilon)$ and three notions of perfect secrecy presented in Definition 2.

It is easy to see that (3) is also represented as $\forall m_{0}, \forall m_{1} \in$ $\mathcal{M}, \forall c \in \mathcal{C}, P_{C \mid M}\left(c \mid m_{0}\right)=P_{C \mid M}\left(c \mid m_{1}\right)^{5}$, which is equivalent to

$$
\forall m_{0}, \forall m_{1} \in \mathcal{M}, d\left(P_{C \mid M}\left(\cdot \mid m_{0}\right), P_{C \mid M}\left(\cdot \mid m_{1}\right)\right)=0 .
$$

Note that (5) implies that cryptograms corresponding to arbitrarily chosen messages $m_{0}$ and $m_{1}$ cannot be statistically distinguished.

We now relax the condition given by (5) using a real number $\varepsilon \in[0,1]$ such that

$$
\forall m_{0}, \forall m_{1} \in \mathcal{M}, d\left(P_{C \mid M}\left(\cdot \mid m_{0}\right), P_{C \mid M}\left(\cdot \mid m_{1}\right)\right) \leq \varepsilon .
$$

According to the definition of variational distance, $d\left(P_{X}, P_{Y}\right) \leq \varepsilon$ can be rewritten as

$\forall f: \mathcal{A} \rightarrow\{0,1\},|\operatorname{Pr}\{f(X)=1\}-\operatorname{Pr}\{f(Y)=1\}| \leq \varepsilon(7)$

and hence, (6) is equivalent to

$$
\begin{aligned}
& \forall m_{0} \in \mathcal{M}, \forall m_{1} \in \mathcal{M}, \forall f: \mathcal{C} \rightarrow\{0,1\}, \\
& \begin{aligned}
\left|\operatorname{Pr}\left\{f(C)=1 \mid M=m_{0}\right\}-\operatorname{Pr}\left\{f(C)=1 \mid M=m_{1}\right\}\right| \\
\leq \varepsilon .
\end{aligned}
\end{aligned}
$$

Note that, (8) is the definiton of computational indistinguishability if the function $f$ is restricted to the family of functions which can be computed in polynomial time $[7,8]$.

\footnotetext{
${ }^{5}$ According to (1) and Theorem 1, perfect secrecy equivalent to $\forall m_{0} \in \mathcal{M}$, $\forall m_{1} \in \mathcal{M}, \forall c \in \mathcal{C}, \operatorname{Pr}\left\{\operatorname{Enc}\left(m_{0}, K\right)=c\right\}=\operatorname{Pr}\left\{\operatorname{Enc}\left(m_{1}, K\right)=c\right\}$, which appears in [6, Proposition 9.3-7.].
} 
Hence, we introduce a security notion of statistical indistinguishability based on (8) as follows.

Definition 3: For a real number $\varepsilon \in[0,1]$, we say that a symmetric key cryptosystem $\Sigma$ is statistically $\varepsilon-$ indistinguishable (IND $(\varepsilon)$-secure, for short) if $\Sigma$ satisfies (6) (and also (8)).

Remark 1: Statistical indistinguishability introduced by Dodis-Smith [4] looks different from Definition 3, but it is easy to show that they are essentially the same.

In the following, we clarify the relation among security notions in Definitions 2 and 3.

Theorem 2: For an arbitrary $\varepsilon \in[0,1]$, a symmetric key cryptosystem $\Sigma$ is $\operatorname{PS}_{C *}(\varepsilon)$-secure iff $\Sigma$ is $\operatorname{IND}(\varepsilon)$-secure.

Proof of Theorem 2: Observe for every $m \in \mathcal{M}$ that

$$
\begin{aligned}
& d\left(P_{C \mid M}(\cdot \mid m), P_{C}(\cdot)\right) \\
& =\frac{1}{2} \sum_{c \in \mathcal{C}}\left|P_{C \mid M}(c \mid m)-\sum_{m^{\prime} \in \mathcal{M}} P_{C \mid M}\left(c \mid m^{\prime}\right) P_{M}\left(m^{\prime}\right)\right| \\
& =\frac{1}{2} \sum_{c \in \mathcal{C}}\left|\sum_{m^{\prime} \in \mathcal{M}} P_{M}\left(m^{\prime}\right)\left\{P_{C \mid M}(c \mid m)-P_{C \mid M}\left(c \mid m^{\prime}\right)\right\}\right|
\end{aligned}
$$

First, we show that $\Sigma$ is $\operatorname{PS}_{C *}(\varepsilon)$-secure if $\Sigma$ is $\operatorname{IND}(\varepsilon)-$ secure. In this case, we assume that $\forall m, \forall m^{\prime} \in \mathcal{M}$, $d\left(P_{C \mid M}(\cdot \mid m), P_{C \mid M}\left(\cdot \mid m^{\prime}\right)\right) \leq \varepsilon$, and hence, from (9) we have

$$
\begin{aligned}
& d\left(P_{C \mid M}(\cdot \mid m), P_{C}(\cdot)\right) \\
& \leq \frac{1}{2} \sum_{m^{\prime} \in \mathcal{M}} P_{M}\left(m^{\prime}\right) \sum_{c \in \mathcal{C}}\left|P_{C \mid M}(c \mid m)-P_{C \mid M}\left(c \mid m^{\prime}\right)\right| \\
& =\sum_{m^{\prime} \in \mathcal{M}} P_{M}\left(m^{\prime}\right) d\left(P_{C \mid M}(\cdot \mid m), P_{C \mid M}\left(\cdot \mid m^{\prime}\right)\right) \\
& \leq \sum_{m^{\prime} \in \mathcal{M}} P_{M}\left(m^{\prime}\right) \varepsilon \\
& =\varepsilon
\end{aligned}
$$

and hence $\Sigma$ is $\operatorname{PS}_{C *}(\varepsilon)$-secure.

We prove the converse. Suppose that $\Sigma$ is $\operatorname{PS}_{C *}(\varepsilon)$-secure. Substitute both $m=m_{0}$ and

$$
P_{M}\left(m^{\prime}\right)=\delta_{m_{1}}\left(m^{\prime}\right) \stackrel{\text { def }}{=} \begin{cases}1, & \text { if } m^{\prime}=m_{1} \\ 0, & \text { otherwise }\end{cases}
$$

into (9). Then, we obtain

$$
\begin{aligned}
d\left(P_{C \mid M}\left(\cdot \mid m_{0}\right), P_{C}(\cdot)\right) & =d\left(P_{C \mid M}\left(\cdot \mid m_{0}\right), P_{C \mid M}\left(\cdot \mid m_{1}\right)\right) \\
& \leq \varepsilon .
\end{aligned}
$$

Hence, $\Sigma$ is $\operatorname{IND}(\varepsilon)$-secure if it is $\operatorname{PS}_{C *}(\varepsilon)$-secure.

The next theorem implies an equivalence between $\operatorname{IND}(\varepsilon)$ and $\operatorname{PS}_{C M}(\varepsilon)$.

Theorem 3: For an arbitrary $\varepsilon \in[0,1]$, a symmetric key cryptosystem $\Sigma$ is $\operatorname{PS}_{C M}(\varepsilon)$-secure if $\Sigma$ is $\operatorname{IND}(\varepsilon)$-secure. Conversely, if $\Sigma$ is $\operatorname{PS}_{C M}(\varepsilon)$-secure, it is $\operatorname{IND}(2 \varepsilon)$-secure.

Proof of Theorem 3: This proof is essentially the same with Theorem 2. Observe that $d\left(P_{C M}, P_{C} P_{M}\right)$ can be calculated as follows:

$$
\begin{aligned}
d & \left(P_{C M}(\cdot, \cdot), P_{C}(\cdot) P_{M}(\cdot)\right) \\
& =\frac{1}{2} \sum_{c \in \mathcal{C}} \sum_{m \in \mathcal{M}}\left|P_{C M}(c, m)-P_{C}(c) P_{M}(m)\right| \\
& =\frac{1}{2} \sum_{c \in \mathcal{C}} \sum_{m \in \mathcal{M}} P_{M}(m)\left|P_{C \mid M}(c \mid m)-P_{C}(c)\right| \\
& =\frac{1}{2} \sum_{c \in \mathcal{C}} \sum_{m \in \mathcal{M}} P_{M}(m) \\
& \times\left|\sum_{m^{\prime} \in \mathcal{M}} P_{M}\left(m^{\prime}\right)\left\{P_{C \mid M}(c \mid m)-P_{C \mid M}\left(c \mid m^{\prime}\right)\right\}\right|
\end{aligned}
$$

We show that $\Sigma$ is $\operatorname{PS}_{C M}(\varepsilon)$-secure if $\Sigma$ is $\operatorname{IND}(\varepsilon)$-secure. In this case, we have from (13) that

$$
\begin{aligned}
& d\left(P_{C M}(\cdot, \cdot), P_{C}(\cdot) P_{M}(\cdot)\right) \\
& \leq \sum_{m, m^{\prime} \in \mathcal{M}} P_{M}(m) P_{M}\left(m^{\prime}\right) d\left(P_{C \mid M}(\cdot \mid m), P_{C \mid M}\left(\cdot \mid m^{\prime}\right)\right) \\
& \leq \varepsilon
\end{aligned}
$$

if $\forall m, \forall m^{\prime} \in \mathcal{M}, d\left(P_{C \mid M}(\cdot \mid m), P_{C \mid M}\left(\cdot \mid m^{\prime}\right)\right) \leq \varepsilon$. Hence, if $\Sigma$ is $\operatorname{IND}(\varepsilon)$-secure, it is also $\operatorname{PS}_{C M}(\varepsilon)$-secure.

Then, suppose that $\Sigma$ is $\operatorname{PS}_{C M}(\varepsilon)$-secure. Then, substituting

$$
P_{M}(m)=\left\{\begin{array}{cl}
1 / 2, & \text { if } m=m_{0} \text { or } m=m_{1} \\
0, & \text { otherwise }
\end{array}\right.
$$

into (13), it follows that

$$
d\left(P_{C M}, P_{C} P_{M}\right)=\frac{1}{2} d\left(P_{C \mid M}\left(\cdot \mid m_{0}\right), P_{C \mid M}\left(\cdot \mid m_{1}\right)\right) \leq \varepsilon .
$$

Hence, $\Sigma$ is $\operatorname{IND}(2 \varepsilon)$-secure if it is $\operatorname{PS}_{C M}(\varepsilon)$-secure.

We have proved that $\operatorname{PS}_{C *}(\varepsilon), \operatorname{PS}_{C M}(\varepsilon)$, and $\operatorname{IND}(\varepsilon)$ are the same security notions. On the other hand, in section $\mathrm{V}$, we show an example that $\mathrm{PS}_{* M}(\varepsilon)$ is stronger security notion than the others in the case of $\varepsilon>0$.

\section{Perfect Secrecy and Semantic Security}

We consider the relation between perfect secrecy and semantic security in information theoretically secure setting. Here, $\operatorname{IND}(\varepsilon)$ also plays a crucial role.

Definition 4 (Statistical semantic security, [3]): For every real number $\varepsilon \in[0,1]$ we say that a symmetric key cryptosystem $\Sigma=\left(P_{K}\right.$, Enc, Dec $)$ is statistically $\varepsilon$-semantic secure (SS $(\varepsilon)$-secure, for short) if, for an arbitrary distribution of a message $P_{M} \in \mathcal{P}(\mathcal{M})$ and for an arbitrary map $f: \mathcal{C} \rightarrow$ $\{0,1\}$, there exists a random variable $G_{f}$ that depends on $f$ but is independent of $M$, so that for every map $h: \mathcal{M} \rightarrow\{0,1\}$, it holds that

$$
\left|\operatorname{Pr}\{f(C)=h(M)\}-\operatorname{Pr}\left\{G_{f}=h(M)\right\}\right| \leq \varepsilon .
$$

Intuitively, Definition 4 implies that a cryptogram $C$ is almost useless to obtain any one bit information of a message $M$, since (17) implies that, in order to guess one bit information $h(M)$ of a message $M$, there is no difference between 
by using a cryptogram $C$ and a map $f$, and by using $f$ only with a random coin.

Remark 2: In [3], $(t, \varepsilon)$-entropic security is defined if a symmetric key cryptosystem $\Sigma$ satisfies Definition 4 for every message with min-entropy $t$, and it is shown that the key length is reduced to $n-t+\omega(\log n)$ bits for $\left(t, n^{-\omega(1)}\right)$ entropic security ${ }^{6}$. Hence, Definition 4 coincides with $(0, \varepsilon)-$ entropic security. Furthermore, it is pointed out in [3] that $(0,0)$-entropic security is equivalent to PS in Definition 1.

We are interested in the relation between PS introduced in Definition 2, and statistical semantic security $\mathrm{SS}(\varepsilon)$ when $\varepsilon>$ 0 . To see this, we show the following relation between $\operatorname{IND}(\varepsilon)$ and $\mathrm{SS}(\varepsilon)$.

Theorem 4: For arbitrary $\varepsilon \in[0,1]$, if a symmetric key cryptosystem $\Sigma$ is IND $(\varepsilon)$-secure, then $\Sigma$ is also SS $(\varepsilon)$-secure. Conversely, if $\Sigma$ is $\operatorname{SS}(\varepsilon)$-secure, then it is also $\operatorname{IND}(4 \varepsilon)-$ secure.

Proof of Theorem 4: First, we prove that $\Sigma$ is SS $(\varepsilon)$-secure if $\Sigma$ is $\operatorname{IND}(\varepsilon)$-secure. This proof is essentially the same with the proof appeared in [8] under computationally secure setting. Let $M^{*}$ be a random variable of a message which is independent of the legitimate message $M$. Then, assume that the random variable $G_{f}$ is generated by $P_{C \mid M}(c \mid m)$ and $M^{*}$, i.e., we define that $G_{f} \stackrel{\text { def }}{=} f\left(C^{*}\right)$ where $P_{C^{*}}(c) \stackrel{\text { def }}{=}$ $\sum_{m_{1}} P_{C \mid M}\left(c \mid m_{1}\right) P_{M^{*}}\left(m_{1}\right)$ for $c \in \mathcal{C}$ and $m \in \mathcal{M}$.

Let us define an indicator function $\mathbb{I}_{f, h}: \mathcal{C} \times \mathcal{M} \rightarrow\{0,1\}$ for maps $f$ and $h$ such that

$$
\mathbb{I}_{f, h}(c, m)= \begin{cases}1, & \text { if } f(c)=h(m) \\ 0, & \text { otherwise }\end{cases}
$$

Then, the left hand side of (17) can be evaluated as

$$
\begin{aligned}
& \left|\operatorname{Pr}\{f(C)=h(M)\}-\operatorname{Pr}\left\{G_{f}=h(M)\right\}\right| \\
& =\left|\operatorname{Pr}\{f(C)=h(M)\}-\operatorname{Pr}\left\{f\left(C^{*}\right)=h(M)\right\}\right| \\
& =\left|\sum_{c, m_{0}} \mathbb{I}_{f, h}\left(c, m_{0}\right)\left\{P_{C M}\left(c, m_{0}\right)-P_{C^{*} M}\left(c, m_{0}\right)\right\}\right| \\
& =\left|\sum_{c, m_{0}} \mathbb{I}_{f, h}\left(c, m_{0}\right) P_{M}\left(m_{0}\right)\left\{P_{C \mid M}\left(c \mid m_{0}\right)-P_{C^{*}}(c)\right\}\right| \\
& =\mid \sum_{m_{0}, m_{1}} P_{M}\left(m_{0}\right) P_{M^{*}}\left(m_{1}\right) \\
& \quad \times \sum_{c} \mathbb{I}_{f, h}\left(c, m_{0}\right)\left\{P_{C \mid M}\left(c \mid m_{0}\right)-P_{C \mid M}\left(c \mid m_{1}\right)\right\} \mid \\
& =\mid \sum_{m_{0}, m_{1}} P_{M}\left(m_{0}\right) P_{M^{*}}\left(m_{1}\right) \times\left\{\operatorname{Pr}\left\{f_{h, m_{0}}(C)=1 \mid M=m_{0}\right\}\right. \\
& \left.-\operatorname{Pr}\left\{f_{h, m_{0}}(C)=1 \mid M=m_{1}\right\}\right\} \mid,
\end{aligned}
$$

where $f_{h, m_{0}}: \mathcal{C} \rightarrow\{0,1\}$ is defined by $f_{h, m_{0}}(c)=1$ iff $\mathbb{I}_{f, h}(c, m)=1$. Then, due to the definition of $\operatorname{IND}(\varepsilon)$ given

$$
{ }^{6} f=\omega(g) \Leftrightarrow \forall \epsilon>0, \exists n_{0}, \forall n \geq n_{0}, g(n) \leq \epsilon f(n) .
$$

by (5), it is easy to see that (19) can be bounded from above by $\sum_{m_{0}, m_{1} \in \mathcal{M}} P_{M}\left(m_{0}\right) P_{M *}\left(m_{1}\right) \cdot \varepsilon=\varepsilon$.

Conversely, we show that $\Sigma$ is $\operatorname{IND}(4 \varepsilon)-$ secure if $\Sigma$ is $\mathrm{SS}(\varepsilon)$-secure. Assuming that a symmetric key cryptosystem $\Sigma$ is $\mathrm{SS}(\varepsilon)$-secure, there exist an arbitrary $f: \mathcal{C} \rightarrow\{0,1\}$ and a random variable $G_{f}$ that depends on $f$ but is independent of $M$, and (17) holds for an arbitrary $h: \mathcal{M} \rightarrow\{0,1\}$.

Now, letting $h$ be a map that always outputs 1 for every $m \in \mathcal{M}$, it holds for arbitrary $f: \mathcal{C} \rightarrow\{0,1\}$ that

$$
\left|\operatorname{Pr}\{f(C)=1\}-\operatorname{Pr}\left\{G_{f}=1\right\}\right| \leq \varepsilon
$$

which is equivalent to

$$
\left|\operatorname{Pr}\{f(C)=0\}-\operatorname{Pr}\left\{G_{f}=0\right\}\right| \leq \varepsilon .
$$

Hence, for $\ell \in\{0,1\}$, it holds that

$$
\operatorname{Pr}\{f(C)=\ell\} \geq \operatorname{Pr}\left\{G_{f}=\ell\right\}-\varepsilon
$$

Multiplying both sides by $\operatorname{Pr}\{h(M)=\ell\} \geq 0$, we have

$$
\begin{aligned}
& \operatorname{Pr}\{f(C)=\ell\} \operatorname{Pr}\{h(M)=\ell\} \\
& \geq\left(\operatorname{Pr}\left\{G_{f}=\ell\right\}-\varepsilon\right) \operatorname{Pr}\{h(M)=\ell\},
\end{aligned}
$$

and hence, it follows that

$$
\begin{aligned}
\sum_{\ell \in\{0,1\}} \operatorname{Pr}\{f(C)=\ell\} \operatorname{Pr}\{h(M)=\ell\} \\
\quad \geq \sum_{\ell \in\{0,1\}}\left(\operatorname{Pr}\left\{G_{f}=\ell\right\}-\varepsilon\right) \operatorname{Pr}\{h(M)=\ell\} \\
\geq \operatorname{Pr}\left\{G_{f}=h(M)\right\}-\varepsilon
\end{aligned}
$$

From (17) we obtain

$$
\begin{aligned}
\operatorname{Pr}\{f(C) & =h(M)\}-\sum_{\ell \in\{0,1\}} \operatorname{Pr}\{f(C)=\ell\} \operatorname{Pr}\{h(M)=\ell\} \\
& \leq \operatorname{Pr}\{f(C)=h(M)\}-\operatorname{Pr}\left\{G_{f}=h(M)\right\}+\varepsilon \\
& \leq 2 \varepsilon .
\end{aligned}
$$

Similarly, by evaluating the upper bound of $\operatorname{Pr}\{f(C)=\ell\}$, $\ell \in\{0,1\}$, we have

$$
\begin{aligned}
& \mid \operatorname{Pr}\{f(C)=h(M)\} \\
& -\sum_{\ell \in\{0,1\}} \operatorname{Pr}\{f(C)=\ell\} \operatorname{Pr}\{h(M)=\ell\} \mid \leq 2 \varepsilon
\end{aligned}
$$

Applying Lemma 1 in Appendix B to this inequality ${ }^{7}$, it holds that

$$
\begin{aligned}
\mid \operatorname{Pr}\{f(C)= & h(M)=1\} \\
& -\operatorname{Pr}\{f(C)=1\} \operatorname{Pr}\{h(M)=1\} \mid \leq \varepsilon .
\end{aligned}
$$

\footnotetext{
${ }^{7}$ Let $X$ and $Y$ in Lemma 1 be $f(C)$ and $h(M)$, respectively.
} 
Since $P_{M} \in \mathcal{P}(\mathcal{M})$ is arbitrary, we set $P_{M}$ in the same way as (15) for arbitrarily fixed $m_{0}, m_{1} \in \mathcal{M}$, and let $h(m)=$ $\delta_{m_{0}}(m)$ which is defined by (11). Then, (27) becomes

$$
\begin{aligned}
& \operatorname{Pr}\left\{M=m_{0}\right\} \mid \operatorname{Pr}\left\{f(C)=1 \mid M=m_{0}\right\} \\
& -\sum_{\ell \in\{0,1\}} \operatorname{Pr}\left\{f(C)=1 \mid M=m_{\ell}\right\} \operatorname{Pr}\left\{M=m_{\ell}\right\} \mid \\
& =\frac{1}{4}\left|\operatorname{Pr}\left\{f(C)=1 \mid M=m_{0}\right\}-\operatorname{Pr}\left\{f(C)=1 \mid M=m_{1}\right\}\right| \\
& \leq \varepsilon .
\end{aligned}
$$

Therefore, $d\left(P_{C \mid M}\left(\cdot \mid m_{0}\right), P_{C \mid M}\left(\cdot \mid m_{1}\right)\right) \leq 4 \varepsilon$ is established for every $m_{0}, m_{1} \in \mathcal{M}$.

\section{A Gap between Perfect Secrecy and Indistinguishability, Semantic Security}

We show an exmaple of a symmetric key cryptosystem $\Sigma$ that is $\operatorname{IND}(\varepsilon)$-secure (and hence, it is also $\operatorname{PS}_{C *}(\varepsilon)$ and $\mathrm{PS}_{C M}(\varepsilon)$-secure) with arbitrarily small $\varepsilon>0$, while it is $\operatorname{PS}_{* M}\left(\varepsilon^{\prime}\right)$-secure with $\varepsilon^{\prime} \geq 1 / 2$. This fact means that $\mathrm{PS}_{* M}(\varepsilon)$ is stronger than the other security notions. We note that $\mathrm{PS}_{* M}(\varepsilon)$ is a straightforward extension of Shannon's perfect secrecy given by (2) in Definition 1 .

Example 1: For an arbitrary even integer $n$, define $\mathcal{C}=$ $\left\{c_{1}, c_{2}, \ldots, c_{n}\right\}$ and $\mathcal{M}=\left\{m_{1}, m_{2}, \ldots, m_{n}\right\}$. Then, consider the following $n \times n$ probability transition matrix corresponding to $P_{C \mid M}$ such that

$$
\begin{aligned}
& \mathbb{P}_{C \mid M} \\
& =\left[\begin{array}{ccccc}
n^{-1}+\delta & n^{-1}-\delta & \cdots & n^{-1}+\delta & n^{-1}-\delta \\
n^{-1}-\delta & n^{-1}+\delta & \cdots & n^{-1}-\delta & n^{-1}+\delta \\
n^{-1} & n^{-1} & \cdots & n^{-1} & n^{-1} \\
\vdots & \vdots & \ddots & \vdots & \vdots \\
n^{-1} & n^{-1} & \cdots & n^{-1} & n^{-1}
\end{array}\right]
\end{aligned}
$$

where $\delta=\varepsilon / 2 \in\left(0, n^{-1}\right]$, and the $(i, j)$ element of $\mathbb{P}_{C \mid M}$ is equal to $P_{C \mid M}\left(c_{i} \mid m_{j}\right)$. From Theorem 1, note that there exists a symmetric key cryptosystem $\Sigma_{\text {ex }}$ corresponding to (29) since it is doubly stochastic.

It is easy to check that $d\left(P_{C \mid M}\left(\cdot \mid m_{i}\right), P_{C \mid M}\left(\cdot \mid m_{j}\right)\right)$ is equal to 0 or $2 \delta(=\varepsilon)$ for each $m_{i}, m_{j} \in \mathcal{M}$. Hence, $\mathbb{P}_{C \mid M}$ realizes a $\operatorname{IND}(\varepsilon)$-secure symmetric key cryptosystem (and hence, it is also $\mathrm{PS}_{C *}(\varepsilon)-$, and $\mathrm{PS}_{C M}(\varepsilon)$-secure).

On the other hand, for uniformly distributed messages, i.e., $P_{M}\left(m_{i}\right)=1 / n, \forall m_{i} \in \mathcal{M}$, it is easy to see that the the transition probability matrix $\mathbb{P}_{M \mid C}$ corresponding to a family of posteriori conditional probability distributions $\left\{P_{M \mid C}(m \mid c)\right\}_{c \in \mathcal{C}, m \in \mathcal{M}}$ corresponds to the transposed matrix of $\mathbb{P}_{C \mid M}$. Hence, in this case

$$
d\left(P_{M \mid C}(\cdot \mid c), P_{M}(\cdot)\right)=\left\{\begin{array}{cl}
n \delta / 2, & \text { if } c=c_{1} \text { or } c_{2} \\
0, & \text { otherwise }
\end{array}\right.
$$

TABLE I

$P_{X Y}$ AND ITS MARGINALS

\begin{tabular}{c|cc|c}
\hline \hline$x \backslash y$ & 0 & 1 & $P_{X}(x)$ \\
\hline 0 & $a$ & $b$ & $a+b$ \\
1 & $c$ & $d$ & $c+d$ \\
\hline$P_{Y}(y)$ & $a+c$ & $b+d$ & 1 \\
\hline \hline
\end{tabular}

which implies that $\Sigma_{\text {ex }}$ is $\mathrm{PS}_{* M}\left(\varepsilon^{\prime}\right)$-secure with ${ }^{8} \varepsilon^{\prime} \geq n \delta / 2$. In particular, $\varepsilon^{\prime} \geq 1 / 2$ for every $n$ if $\varepsilon=2 / n(=2 \delta)$ which can be arbitrarily small for sufficiently large $n$.

In this example, the symmetric key cryptosystem $\Sigma_{\text {ex }}$ given by (29) violates $d\left(P_{M \mid C}(\cdot \mid c), P_{M}(\cdot)\right) \leq \varepsilon$ with the negligibly small probability $\operatorname{Pr}\left\{C=c_{1} \vee C=c_{2}\right\}=2 / n$ if $P_{M}$ is uniform and $n$ is sufficiently large, although it is required by $\mathrm{PS}_{* M}(\varepsilon)$-security to satisfy $d\left(P_{M \mid C}(\cdot \mid c), P_{M}(\cdot)\right) \leq \varepsilon$ for every $c \in \mathcal{C}$. On the other hand, $\Sigma_{\text {ex }}$ is still considered to be secure under the other security notions since they focus on the probability distribution of $C$ and the probability that such insecure cryptograms are output is negligible.

\section{ACKNOWLEDGEMENT}

The authors would like to thank Prof. Hideki Imai in Chuo University, Prof. Ryutaroh Matsumoto in Tokyo Institute of Technology, and Mr. Yusuke Sakai in University of ElectroCommunications for their helpful comments. The work of the first author, M. Iwamoto is partially supported by the MEXT Grant-in-Aid for Young Scientists (B) No. 20760236.

\section{REFERENCES}

[1] C. E. Shannon, "Communication theory of secrecy systems," Bell Tech J., vol. 28 , pp. $656-715$, Oct. 1949

[2] G. S. Vernam, "Cipher printing telegraph systems for secret wire and radio telegraphic communications," J. of American Institute for Electrical Engineering, vol. 45, pp. 109-115, 1926.

[3] A. Russell and H. Wang, "How to foll an unbounded adversary with a short key," IEEE Trans. Information Theory, pp. 1330-1140, 2006. Preliminary version: EUROCRYPT 2002, LNCS 2332, Springer-Verlag, pp.133-148, 2002.

[4] Y. Dodis and A. Smith, "Entropic security and the encryption of high entropy messages," TCC 2005, pp. 556-577, 2005. Full version: IACR Cryptology ePrint Archive, report 2004/219, at http://eprint.iacr.org/2004/219/.

[5] H. Krawczyk, "LFSR-based hashing and authentication," Advances in Cryptology-CRYPTO'94, LNCS 839, Springer-Verlag, pp. 129-139, 1994.

[6] H. Delfs and H. Knebel, Introduction to Cryptography, Principles and Applications. Information Security and Cryptography Texts and Monographs, Springer-Verlag, second ed., 2001.

[7] S. Goldwasser and S. Micali, "Probabilistic encryption," Journal of Computer and System Sciences, vol. 28, no. 2, pp. 270-299, 1984.

[8] O. Goldreich, Foundations of Cryptography Volume I Basic Tools. Springer-Verlag, 2001

\section{APPENDIX}

\section{A. Proof of Theorem 1}

Observe that a random variable $C$ of a cryptogram is obtained by $C=\operatorname{Enc}(M, K)$, where $M$ and $K$ are independent

\footnotetext{
${ }^{8}$ Note that $d\left(P_{M \mid C}(\cdot \mid c), P_{C}(\cdot)\right) \leq \varepsilon$ holds for every $P_{M}$ to ensure $\mathrm{PS}_{* M}(\varepsilon)$-secure cryptosystems.
} 
random variables of a message and a key, respectively, and Enc: $\mathcal{M} \times \mathcal{K} \rightarrow \mathcal{C}$ is a deterministic map of encryption. Hence, the joint probability distribution $P_{C M}(c, m)$ of a cryptogram and a message can be represented as

$$
\begin{aligned}
P_{C M}(c, m) & =\operatorname{Pr}\{C=c, M=m\} \\
& =\operatorname{Pr}\{\operatorname{Enc}(M, K)=c, M=m\} \\
& =\sum_{k: \operatorname{Enc}(m, k)=c} P_{M K}(m, k) \\
& \stackrel{(*)}{=} P_{M}(m) \sum_{k: \operatorname{Enc}(m, k)=c} P_{K}(k) \\
& =P_{M}(m) \operatorname{Pr}\{\operatorname{Enc}(m, K)=c\},
\end{aligned}
$$

where the marked equality holds since $M$ and $K$ are independent. Hence, we have (1).

In what follows, we consider the case of $|\mathcal{M}|=|\mathcal{C}|$. In this case, if $k \in \mathcal{K}$ is fixed, there exists a bijection $\pi_{k}: \mathcal{M} \rightarrow$ $\mathcal{C}$ since every cryptogram $c \in \mathcal{C}$ can be uniquely decrypted by $k \in \mathcal{K}$. Hence, for each $k \in \mathcal{K}$, let $\Pi_{k} \in\{0,1\}^{n \times n}$ be a permutation matrix which corresponds to the bijection $\pi_{k}$. Then, it is easy to see that the probability transition matrix induced by Enc and $K$ can be represented as

$$
\mathbb{P}_{C \mid M}=\sum_{k \in \mathcal{K}} P_{K}(k) \Pi_{k},
$$

which is doubly stochastic. Conversely, due to Birkoff-von Neumann Theorem, there exists a pair of $P_{K}(k)$ and $\Pi_{k}, k \in$ $\mathcal{K}$, satisfying (32) if $\mathbb{P}_{C \mid M}$ is doubly stochastic.

\section{B. Lemma in Proof of Theorem 4}

In proof of Theorem 4, we use the following lemma:

Lemma 1: For two binary random variables $X$ and $Y$ over a set $\{0,1\}$, and for $\varepsilon \in[0,1]$, the following two inequalities are equivalent:

$$
\begin{gathered}
\left|\operatorname{Pr}\{X=Y\}-\sum_{\ell \in\{0,1\}} \operatorname{Pr}\{X=\ell\} \operatorname{Pr}\{Y=\ell\}\right| \leq \varepsilon \\
|\operatorname{Pr}\{X=Y=\ell\}-\operatorname{Pr}\{X=\ell\} \operatorname{Pr}\{Y=\ell\}| \leq \frac{\varepsilon}{2}, \\
\ell \in\{0,1\}
\end{gathered}
$$

We show that (33) $\Rightarrow$ (34) since (34) $\Rightarrow$ (33) is obvious. Letting $P_{X Y}(x, y), x, y \in\{0,1\}$ be a joint probability distribution of $X$ and $Y$ given by TABLE I, (33) is equivalent to

$$
|a+d-(a+b)(a+c)-(c+d)(b+d)| \leq \varepsilon .
$$

Since it holds that $a+b+c+d=1$, (35) becomes $|a d-b c| \leq$ $\varepsilon / 2$. Furthermore, using $a+b+c+d=1$ again, we have

$\left|P_{X Y}(0,0)-P_{X}(0) P_{Y}(0)\right|=|a-(a+b)(a+c)| \leq \frac{\varepsilon}{2}(36)$
$\left|P_{X Y}(1,1)-P_{X}(1) P_{Y}(1)\right|=|d-(c+d)(b+d)| \leq \frac{\varepsilon}{2}$ (37) which implies (33).

Remark 3: Note that (33) $\Rightarrow$ (34) does not generally hold if $X$ and $Y$ are not binary random variables. 